\title{
Efecto del ajuste socioemocional en el rendimiento y las competencias académicas en el contexto escolar: Estudio comparativo
}

\author{
Ma Ángeles Fernández-Vilar*, José Antonio Carranza-Carnicero, Manuel Ato-García
}

Universidad de Murcia (España)

\begin{abstract}
Resumen: El propósito del trabajo fue estudiar el ajuste socioemocional y los problemas de conducta, en niños de 11 años de edad, y su relación con el rendimiento y las competencias académicas en Educación Primaria. Profesores y madres cumplimentaron la Batería de Socialización (BAS; Silva y Martorell, 1999) en una muestra de 49 niños, pertenecientes a un estudio longitudinal más amplio. El rendimiento académico se evaluó a través de las calificaciones del profesor y de una prueba estandarizada. Las competencias académicas se midieron a través del cuestionario Health Resources Inventory (HRI; Keogh, Juvonen, y Bernheimer, 1989). También se controló el efecto de la inteligencia mediante el CI verbal de los niños. Nuestros resultados indican acuerdo entre profesores y madres en la evaluación del ajuste socioemocional positivo y del retraimiento de los niños, pero señalan discrepancias en la relación que los diferentes aspectos del ajuste socioemocional mantienen con el rendimiento y con las competencias académicas. Estos datos sugieren que las madres tienen una visión cercana a la del profesor en relación con los comportamientos socioemocionales de los niños, pero únicamente la visión del profesor guarda relación con la ejecución académica del niño. Además, tanto la inteligencia como el ajuste socioemocional son necesarios para explicar el rendimiento y las competencias académicas.

Palabras clave: Ajuste socioemocional; problemas de comportamiento;
\end{abstract} rendimiento académico; competencias académicas; educación primaria.
Title: Effect of socio-emotional adjustment on the school achievement and academic competence in school: Cross-informant study.

Abstract: The aim of the current study was to examine the role of socioemotional adjustment and behavioral problems in eleven years old children; as well as their relationships with school achievement and academic competence in Primary School. Teachers and mothers completed the Social Battery (BAS, Silva, \& Martorell, 1999) in a sample of 49 children that take part in a longitudinal study. School achievement was measured by standardized test and qualifications in reading and math. The academic competence was evaluated by teachers with a questionnaire (HRI, Keogh, Juvonen, \& Bernheimer, 1989). The effect of intelligence was controlled by the IQ. These data showed that intelligence with socio-emotional adjustment were both of them necessary to explain school achievement and academic competences. Also, results showed agreement between mothers and teachers in positive social adjustment and withdrawing. There were discrepancies as to the effect of socio-emotional adjustment on academic competence and school achievement. These data suggest that mothers and teachers agree in their view of children's socio-emotional behavior, but only teachers' views were related to school achievement.

Key words: Socio-emotional adjustment; Behavioral problems; School achievement; Academic competence; Primary School.
El estudio de las características psicosociales y psicopatológicas en niños y adolescentes es un tópico que suscita gran interés en nuestros días. Burt, Obradovicc, Long y Masten (2008), revisan la interacción existente entre la competencia social y la psicopatología en los últimos 20 años, poniendo de relieve que el ajuste social se relaciona de forma positiva con el desarrollo saludable del niño (Parker, Rubin, Erath, Wojslawowicz, y Buskirk, 2006; Rubin, Bukowski, y Parker, 2006), hasta el punto que se ha considerado como un factor protector contra los problemas de conducta (Carreras, Braza y Braza, 2001). En otras investigaciones se señala también la contribución del ajuste social positivo a la competencia académica de los niños (DiPema y Elliot, 2002; DiPerna, Volpe y Elliot, 2002; Demaray y Malecki, 2002), destacando su importante influencia incluso en situaciones de victimización en la escuela (Tu, Erath y Flanagan, 2012). A este respecto, se ha encontrado que las experiencias de victimización en los niños y adolescentes se asocian con un efecto negativo sobre la ejecución académica (Nakamoto y Schwartz, 2010), mientras que la capacidad de tener amistades positivas se asocia con una mejor competencia académica e incluso puede llegar a convertirse en un factor protector contra el efecto negativo que la situaciones de victimización en la escuela tienen sobre la competencia académica (Palmen, Vermande, Dekovic y van Aken, 2011; Schwartz,

* Dirección para correspondencia [Correspondence address]: $\mathrm{M}^{\mathrm{a}}$ Ángeles Fernández Vilar. Dept ${ }^{\circ}$ de Psicología Evolutiva y de la Educación. Universidad de Murcia. Campus de Espinardo. 30100 Murcia. E-mail: mafvilar@um.es
Gorman, Dodge, Pettit y Bates, 2008). Por otro lado, también se ha encontrado que el ajuste positivo presenta un mayor valor predictivo del rendimiento académico que el que tienen los problemas de conducta e incluso la misma competencia académica (Ray y Elliot, 2006).

El ajuste social también se relaciona de forma negativa con la psicopatología desde edades muy tempranas (BriggsGowan y Carter, 2008). En este sentido, se ha observado que cambios en la competencia social pueden llegar a predecir cambios en los síntomas depresivos (Gazelle y Ladd, 2003; Masten, Burk y Coatsworth, 2006; Parker et al., 2006). También se ha encontrado que durante la niñez el rechazo social de los iguales se asocia con comportamientos agresivos (Dishion y Patterson, 2006; Hinshaw y Lee, 2003) llegando a predecirlos durante la niñez (Campbell, Shaw y Gillion, 2000) y adolescencia (Coie y Dodge, 1998). Además, los déficits en la competencia socioemocional pueden conducir al desarrollo de problemas en diferentes áreas como la educativa, psicosocial, y laboral (Elliot y Gresham, 2008; Zubeidat, Fernández-Parra, Ortega, Vallejo y Sierra, 2009). Sin embargo, no todos los problemas de adaptación socioemocional que aparecen durante la niñez y adolescencia tienen las mismas repercusiones evolutivas (Sandoval, Lemos y Vallejo, 2006). Por una parte, los problemas externalizantes, fundamentalmente la agresión, tienden a asociarse con resultados negativos a largo plazo en forma de fracaso escolar, comportamientos escolares problemáticos en la niñez y adolescencia y la aparición de conductas delictivas posteriores (Briggs-Gowan y Carter, 2008; Preddy y Fite, 2012; Putallaz, Grimes, Foster, Kuppersmidt, Coie y Dearing, 2007). Por 
otra parte, los desórdenes internalizantes (fundamentalmente ansiedad y depresión) aunque también reflejan secuelas negativas en el rendimiento académico del niño, resultan de menor intensidad y con una menor proyección en el tiempo, en relación a los problemas externalizantes (Ansary y Luthar, 2009; Galaif, Sussman, Chou yWills, 2003; Burt y Roisman, 2010; Masten, Roisman, Long, Burt, Obradovic, Riley, Boelcke-Stennes y Tellegen, 2005). Además, dicha influencia negativa de los síntomas internalizantes sobre la ejecución escolar se incrementa a mayores niveles de estrés experimentados por el niño (Alva y De los Reyes, 1999; Cunningham, Hurley, Foney y Hayes, 2002).

En las investigaciones con niños y adolescentes, las discrepancias entre informantes constituye un fenómeno robusto aunque insuficientemente comprendido (Hartley, Zakriski y Wright, 2011). Numerosos estudios han investigado las características del niño y de los informantes que predicen las discrepancias, pero todavía hoy, nuestra comprensión de los mecanismos subyacentes permanece incompleta y suscita un vivo debate (ver Achenbach, 2006; Gresham, Elliot, Cook, Vance y Kettler, 2010; Karver, 2006). A este respecto, hay consenso en aceptar que los datos deben ser recogidos de múltiples informantes; sin embargo, se observa menos entendimiento respecto a cómo utilizar la información procedente de las distintas fuentes de evaluación. Como recogen Dirks, Boyle y Georgiades (2011), a menudo, los investigadores utilizan técnicas que enfatizan el acuerdo interobservadores como una combinación de datos de distintos evaluadores, donde el grado de acuerdo entre los mismos se considera que acentúa el valor del rasgo de conducta analizado, mientras que las discrepancias se consideran como "ruido" o "sesgos" y son explicados atendiendo a los diferentes niveles de validez de las fuentes utilizadas (Kraemer, Measelle, Ablow, Essex, Boyce y Kupfer, 2003). En esta línea, se ha argumentado que el grado de acuerdo entre los informantes puede estar relacionado con la "saliencia" de las conductas analizadas. El acuerdo tiende a ser mayor ante comportamientos que son fáciles de observar o bien generan daños de manera crónica y evidente (peleas, escapadas del hogar, llanto). Mientras que el acuerdo tiende a ser mucho menor ante síntomas de tipo internalizante (evaluaciones negativas o la ideación suicida), al ser más difíciles de observar (Comer y Kendall, 2004; Foley, Rutter, Angold, Pickles, Maes y Silberg, 2005; Kemper, Gerhardstein, Repper y Kistner, 2003).

No obstante, como ya señalaba Karver (2006) y más recientemente Dirks y colaboradores (Dirks et al., 2011), varias líneas de evidencia sugieren que las discrepancias entre las evaluaciones de padres y profesores reflejan algo más que una mera medida de error o problemas de validez entre sus informadores. En primer lugar, porque las evaluaciones procedentes de diferentes informantes se ha demostrado que tienen una alta fiabilidad (Drabick, Gadow y Lonely, 2007). En segundo lugar, porque se ha demostrado la importancia de tener en cuenta la especificidad de las situaciones en las que se manifiestan las conductas analizadas (fundamental- mente las consideradas como problemáticas), ya que pueden estar reflejando variaciones significativas del comportamiento de los niños según el contexto en que se analicen (Frank, van Egeren, Fortier y Chase, 2000), así como diferentes expectativas y juicios por parte de los evaluadores. En este sentido, se ha argumentado que los padres se ven expuestos al comportamiento de sus hijos durante mayores períodos de tiempo, y generalmente suelen tener una experiencia limitada con otros niños. En cambio, la experiencia que los profesores tienen con la conducta de los niños es mucho más restringida en el tiempo, aunque la pueden contrastar con un número mucho mayor y variado de comportamientos de otros niños de edad similar (De los Reyes, Henry, Tolan y Wakschlag, 2009; Drabick, Gadow y Lonely, 2008; Guion, Mrug y Windle, 2009; Hartley et al., 2011). En tercer lugar, las discrepancias entre padres y profesores suelen ser más acusadas en relación a los síntomas internalizantes y menores con los síntomas externalizantes (Berg-Nielsen, Solheim, Belsky y Wichstrom, 2011; Ferdinand, Hoogerheide, Van der Ende, Heigmens, Visser, Koot, Kasius et al., 2003). Las diferentes manifestaciones en estos síntomas podría estar mediatizada por la naturaleza de los contextos en los que se evalúan, así como por los diferentes grados de saliencia e importancia concedida por unos y otros evaluadores (ver Collishaw, Goodman, Ford, Rabe-Hesketh y Pickles, 2009 para una revisión).

Recientemente De los Reyes y colaboradores (De los Reyes, Youngstrom, Pabón, Youngstrom, Feeny, Findling y 2011), han llamado la atención sobre la necesidad de tener en cuenta las propiedades psicométricas de los análisis estadísticos utilizados para el cálculo de los niveles de acuerdo entre evaluadores. Han encontrado que los niveles de acuerdo varían en función de cómo se calcula el grado de acuerdo interobservador (global) y la manera en que se analizan las discrepancias en función de su magnitud (qué cantidad de acuerdo/discrepancia hay) y de su dirección (qué evaluación resulta mayor).

En el análisis del efecto del ajuste socioemocional en el rendimiento y las competencias académicas, también se ha controlado el efecto del CI. En investigaciones previas se ha puesto de manifiesto que el CI ejerce un efecto directo sobre el funcionamiento académico de los niños, tanto en cuanto a su rendimiento como sus competencias (Caspi, Wright, Moffit y Silva, 1998). Por otra parte, también se observan asociaciones del CI con el ajuste conductual del niño, fundamentalmente con los problemas de conducta y los desórdenes por déficit de atención (Bierdeman, Faraone, Milberger, Guite, Mick, Chen, Mennin et al., 1996; Rapport, Scalan y Denney, 1999). A este respecto, se ha llegado a hipotetizar que los problemas de conducta externalizantes pudieran estar mediando la relación de la inteligencia con posteriores resultados académicos de los niños. Así, se ha encontrado una clara tendencia a que el incremento en el CI vaya asociado a menores índices de problemas de conducta tanto de de tipo externalizante como de ansiedad. Fergusson, Horwood y Ridder (2005) ofrecen tres posibles explicaciones a la tem- 
prana asociación encontrada entre los problemas de conducta y el CI. En primer lugar, sugieren que esta asociación podría deberse a que comparten unos procesos familiares, sociales y genéticos comunes. En el sentido que los factores que se conoce que se asocian con el CI (estatus socioeconómico, nivel educativo de los padres, funcionamiento familiar, etc) son bastante similares a los que se asocian con los problemas de conducta (Farrington, 1995). Una segunda explicación es que una de las consecuencias de una baja inteligencia es tener una mayor predisposición a los problemas de conducta (Jensen, 1998). La tercera explicación es que los problemas de conducta pueden influir la medida de la inteligencia, dado que los individuos que son propensos a manifestar conductas difíciles inadaptadas o desafiantes tienden a rendir peor en las pruebas de inteligencia (Goodman, Simonoff y Stevenson, 1995).

Como se puede observar, el ajuste socio-emocional del niño guarda una estrecha relación con su funcionamiento académico, al tiempo que se pone de manifiesto la existencia de discrepancias entre los evaluadores en las interpretaciones de las relaciones entre la naturaleza de las caractersiticas socio-emocionales y de los contextos considerados para realizar la evaluación. En esta dirección planteamos los objetivos de nuestro trabajo. En primer lugar, analizar el grado de acuerdo entre madres y profesores respecto a la evaluación de la adaptación socioemocional de los niños. En segundo lugar, analizar qué aspectos del ajuste socioemocional resultan más significativos para las madres y para los profesores a la hora de predecir el rendimiento y la competencia académica de los niños.

\section{Método}

\section{Participantes}

Los sujetos que colaboraron en este trabajo integran un estudio longitudinal más amplio sobre el desarrollo del temperamento en la infancia. Inicialmente la muestra estuvo compuesta por 60 familias procedentes de diferentes localidades de la Región de Murcia (España). En el momento de la realización de este estudio contamos con 49 sujetos (22 niños y 27 niñas), y sus madres pertenecientes a un nivel socio-económico medio. Aproximadamente el 17\% de las madres tenía estudios universitarios, el 41\% había completado estudios secundarios, y un $42 \%$ tenía estudios primarios. En el momento del presente estudio, los niños habían cumplido 11 años de edad y estaban cursando $6^{\circ}$ de Educación Primaria. Los centros en los que se encontraban escolarizados dichos niños sumaban un total de 37 centros (25 centros públicos y 12 centros concertados) y estaban distribuidos por diferentes comarcas de la Región de Murcia. Los tutores de cada uno de estos niños también participaron en el estudio. Ninguno de los niños tenía diagnosticados problemas de aprendizaje.

\section{Instrumentos}

Ajuste socioemocional. Como medida de la adaptación socioemocional del niño se utilizó la Batería de Socialización de Silva y Martorell (1999), en su versión para profesores $(B A S$ 1) y en la de padres $(B A S-2)$. Permite obtener una puntuación de ajuste socioemocional positivo y tres tipos de problemas de conducta (agresividad, retraimiento, ansiedad).

Rendimiento escolar. Para la evaluación del rendimiento académico de los niños se tomaron dos tipos de medidas al final del curso académico (junio). Una prueba de rendimiento en Lectura y Matemáticas, basada en los contenidos curriculares básicos en dichas materias de los alumnos de $3^{\circ}$ Ciclo de Educación Primaria. También se utilizó la calificación final del profesor en las áreas de Lectura y Matemáticas. Las notas obtenidas se estandarizaron por grupo y por centro escolar. Las correlaciones entre las medidas de rendimiento oscilaban entre $r=.60$ y $r=.82$.

Competencia académica. Como medida de las competencias académicas de los niños los profesores cumplimentaron a final de curso la versión española del cuestionario Health Resources Inventory (HRI, Keogh, Juvonen y Bernheimer, 1989), que evalúa la percepción que el profesor tiene del comportamiento del alumno en el contexto escolar mediante cinco escalas. En nuestra muestra, dichas escalas obtuvieron adecuados índices de frabilidad. Seguimiento de las reglas $(\alpha=$ $.84)$, Responsabilidad en el aprendizaje ( $\alpha=.94)$, Sociabilidad $(\alpha=.88)$, Seguridad $(\alpha=.89)$, y Tolerancia a la frustración $(\alpha=.90)$.

Inteligencia. Se tomó una medida de cociente intelectual de cada niño mediante la prueba de inteligencia WISC-R (Wechsler, 2000). Específicamente, se administraron de forma individualizada las escalas verbales de la prueba para calcular el CI Verbal.

\section{Resultados}

En respuesta a nuestro primer objetivo, de analizar el grado de concordancia entre las dos versiones del cuestionario de adaptación socioemocional, se utilizaron coeficientes de correlación intraclase, y se obtuvieron valores estadísticamente diferentes de cero en el caso de ajuste positivo $\left(R_{\mathrm{I}}=.51\right) \mathrm{y}$ retraimiento $\left(R_{I}=.41\right)$. Pero no se hallaron diferencias entre versiones en agresividad $\left(R_{I}=.25\right)$ y en ansiedad $\left(R_{I}=-.01\right)$.

Para dar respuesta al segundo objetivo se siguieron varios pasos. En primer lugar, se realizó un análisis descriptivo. Mediante correlaciones de Pearson se detectaron qué variables de ajuste socioemocional se asociaban con cada una de las variables educativas analizadas (rendimiento y competencia académica). También se introdujeron las correlaciones del CI Verbal con cada una de dichas variables educativas (véase Tabla 1 para un análisis de todas las correlaciones obtenidas).

En segundo lugar, se realizaron análisis de regresión jerárquicos para analizar el impacto de las evaluaciones del ajuste socioemocional con en el rendimiento escolar. En to- 
dos los análisis, para controlar el posible efecto de multicolinealidad que el CI Verbal podría tener se introdujo junto a las variables predictoras (medidas de ajuste socioemocional).

Desde la perspectiva del profesor se encuentra que el ajuste socioemocional positivo, junto con el CI verbal, actúa como predictor del rendimiento evaluado por el profesor, aunque no así del rendimiento evaluado mediante prueba objetiva. Los problemas de agresividad únicamente predicen el rendimiento en matemáticas evaluado por el profesor. Los problemas relacionados con la apatía-retraimiento, junto con el CI verbal, predicen una peor ejecución en todas las medidas de rendimiento registradas. Los problemas de ansiedad predicen, junto con el CI verbal, el rendimiento en matemáticas evaluado mediante prueba objetiva (véase Tabla 2 para un resumen de los análisis de regresión entre las medidas de ajuste socioemocional procedentes del profesor y las medidas de rendimiento académico).

Sin embargo, cuando la evaluación del ajuste socioemocional procede de las madres, ninguna de las medidas de dicho ajuste (ajuste positivo, agresividad, retraimiento, ansiedad) predicen el rendimiento académico (véase Tabla 3 para un resumen de los análisis de regresión entre las medidas de ajuste socioemocional procedente de las madres y las medidas de rendimiento académico).

Tabla 1. Correlaciones Pearson entre la Inteligencia, Rendimiento, Competencia académica y Ajuste socioemocional $(N=49)$.

\begin{tabular}{|c|c|c|c|c|c|c|c|c|c|c|c|c|c|c|c|c|c|c|}
\hline & 1 & 2 & 3 & 4 & 5 & 6 & 7 & 8 & 9 & 10 & 11 & 12 & 13 & 14 & 15 & 16 & 17 & 18 \\
\hline \multicolumn{19}{|l|}{$\overline{1}$} \\
\hline 2 & $.47^{* *}$ & & & & & & & & & & & & & & & & & \\
\hline 3 & $.46^{* *}$ & $.77 * *$ & & & & & & & & & & & & & & & & \\
\hline 4 & $.43^{* *}$ & $.49^{* *}$ & $.45^{* *}$ & & & & & & & & & & & & & & & \\
\hline 5 & $.36^{* *}$ & $.56^{* *}$ & $.67^{* *}$ & $.62 * *$ & & & & & & & & & & & & & & \\
\hline 6 & .08 & $.41 * *$ & $.42^{* *}$ & .28 & .28 & & & & & & & & & & & & & \\
\hline 7 & $.45^{* *}$ & $.82^{* *}$ & $.75^{* *}$ & $.47^{* *}$ & $.51 * *$ & $.54^{*}$ & & & & & & & & & & & & \\
\hline 8 & $.55^{* *}$ & $.42^{* *}$ & $.33^{*}$ & .24 & .27 & .04 & $.41^{* *}$ & & & & & & & & & & & \\
\hline 9 & $.29+$ & $.68^{* *}$ & $.63^{* *}$ & $.48^{* *}$ & $.53^{* *}$ & $.62^{* *}$ & $.77^{* *}$ & $.38^{*}$ & & & & & & & & & & \\
\hline 10 & .22 & $.49 * *$ & $.49 * *$ & $.32^{*}$ & $.39 * *$ & $.66^{* *}$ & $.63^{* *}$ & .23 & $.79 * *$ & & & & & & & & & \\
\hline 11 & .20 & $.55^{* *}$ & $.50^{* *}$ & $.34 *$ & $.33^{*}$ & $.69^{* *}$ & $.72^{* *}$ & $.33^{*}$ & $.85^{* *}$ & $.75^{* *}$ & & & & & & & & \\
\hline 12 & .00 & -.23 & $-.35^{*}$ & -.14 & -.13 & $-.77 * *$ & $-.36^{*}$ & $.32 *$ & $-.47 * *$ & $-.53^{* *}$ & $-.53 * *$ & & & & & & & \\
\hline 13 & -.17 & $-.59 * *$ & $-.44 * *$ & $-.37 *$ & $-.56^{*}$ & -.18 & $-.48 * *$ & $-.37 *$ & $-.64 * *$ & -.28 & $-.45^{* *}$ & .07 & & & & & & \\
\hline 14 & .06 & -.22 & -.15 & -.12 & -.29 & .15 & -.06 & -.27 & -.11 & .12 & .04 & -.16 & $.55^{* *}$ & & & & & \\
\hline 15 & .10 & .24 & .12 & -.01 & -.02 & $.30+$ & $.40^{*}$ & .13 & $.46^{* *}$ & $.50^{* *}$ & $.51 * *$ & -.28 & -.25 & .14 & & & & \\
\hline 16 & -.11 & -.00 & .01 & -.13 & -.04 & -.17 & -.13 & -.06 & $-.32 *$ & $-.38 *$ & $-.33^{*}$ & .24 & .14 & -.19 & $-.63 * *$ & & & \\
\hline 17 & $-.31 *$ & -.10 & -.05 & -.10 & -.16 & .14 & -.18 & $-.37^{*}$ & -.28 & -.19 & -.18 & -.12 & $.41^{* *}$ & .07 & $-.57 * *$ & $.65^{* *}$ & & \\
\hline 18 & $-.35 *$ & -.05 & -.03 & -.10 & -.04 & .07 & -.12 & $-46^{* *}$ & -.19 & -.24 & -.14 & -.08 & .18 & -.02 & $-.37 *$ & $.40^{* *}$ & $.67 * *$ & \\
\hline
\end{tabular}

Nota: Inteligencia ( $1=$ CI verbal). Rendimiento ( $=$ Lectura profesor; $3=$ Matemáticas profesor; 4 = Lectura en test; $5=$ Matemáticas en test). Competencia académica ( 6 = Seguimiento de reglas; $7=$ Responsabilidad en el aprendizaje; $8=$ Seguridad; $9=$ Sociabilidad; $10=$ Tolerancia a la frustración). Ajuste socioemocional profesor $(11=$ Ajuste positivo profesor; $12=$ Agresividad profesor; $13=$ Retraimiento; $14=$ Ansiedad profesor $)$. Ajuste socioemocional madre (15 = Ajuste positivo madre; $16=$ Agresividad madre; $17=$ Retraimiento madre; $18=$ Ansiedad madre).

$*(p<.05), * *(p<.001)$.

Tabla 2. Resumen de los análisis de regresión jerárquicos entre competencia socioemocional evaluada por el profesor y rendimiento académico $(N$ = 49).

\begin{tabular}{|c|c|c|c|c|c|c|c|}
\hline Criterio & Modelo & $\mathrm{R}^{2}$ & $F$ & $B$ & SE B & $\beta$ & $t$ \\
\hline \multicolumn{8}{|c|}{ Ajuste Positivo Profesor (AJP) } \\
\hline \multirow[t]{4}{*}{ LP } & & & & & & & \\
\hline & AJP & .29 & $18.31 * *$ & .08 & .02 & .55 & $4.28^{* *}$ \\
\hline & AJP & .42 & $16.59 * *$ & .07 & .02 & .47 & $3.97 * *$ \\
\hline & CIv & & & .03 & .01 & .39 & $3.26 * *$ \\
\hline \multicolumn{8}{|l|}{ MP } \\
\hline & AJP & .24 & $14.32 * *$ & .08 & .02 & .50 & $3.78^{* *}$ \\
\hline & AJP & .37 & $13.68 * *$ & .06 & .02 & .42 & $3.43^{* *}$ \\
\hline & CIv & & & .04 & .01 & .39 & $3.16^{* *}$ \\
\hline \multicolumn{8}{|l|}{$\mathrm{LT}$} \\
\hline & $\mathrm{CIv}$ & .17 & $9.74 * *$ & .08 & .02 & .43 & $3.12^{* *}$ \\
\hline \multirow{2}{*}{\multicolumn{8}{|c|}{$\begin{array}{l}\quad 6.45^{*} \\
\text { resividad Profes }\end{array}$}} \\
\hline & & & & & & & \\
\hline \multicolumn{8}{|l|}{ LP } \\
\hline & $\mathrm{CIv}$ & .22 & $12.85^{* *}$ & .04 & .01 & .48 & $3.58 * *$ \\
\hline \multicolumn{8}{|l|}{ MP } \\
\hline & $\mathrm{CIv}$ & .21 & $12.39 * *$ & .05 & .01 & .48 & $3.52^{* *}$ \\
\hline & $\mathrm{CIv}$ & .32 & $11.24 * *$ & .05 & .01 & .48 & $3.81 * *$ \\
\hline & AGR & & & -.05 & .02 & -.35 & $-2.83 * *$ \\
\hline \multicolumn{8}{|l|}{ LT } \\
\hline & $\mathrm{CIv}$ & .17 & $9.74 * *$ & .07 & .02 & .43 & $3.12^{* *}$ \\
\hline
\end{tabular}




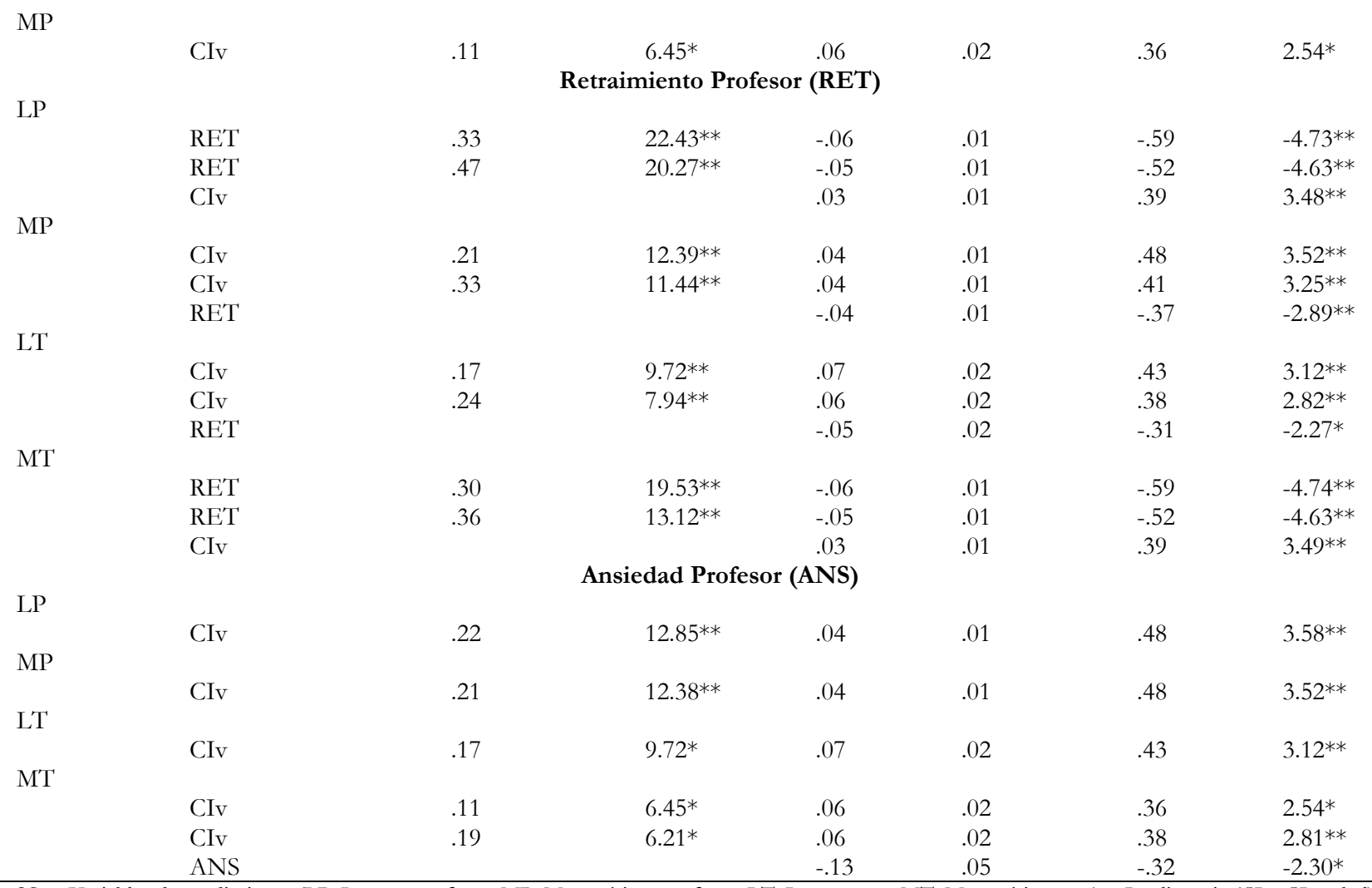

Nota: Variables de rendimiento (LP: Lectura profesor; MP: Matemáticas profesor; LT: Lectura test; MT: Matemáticas test). Inteligencia (CIv: CI verbal). ${ }^{*}(p$ $<.05), * *(p<.001)$

Tabla 3. Análisis de regresión entre ajuste socioemocional evaluado por la madre y rendimiento $(\mathrm{N}=49)$

\begin{tabular}{|c|c|c|c|c|c|c|c|}
\hline Criterio & Modelo & $R^{2}$ & $F$ & $B$ & SE B & $\bar{\beta}$ & $t$ \\
\hline \multicolumn{8}{|c|}{ Ajuste Positivo Madre } \\
\hline $\mathrm{LP}$ & $\mathrm{CIv}$ & .20 & $12.36^{* *}$ & .04 & .01 & .47 & $3.51 * *$ \\
\hline MP & $\mathrm{CIv}$ & .20 & $11.77 * *$ & .04 & .01 & .46 & $3.43 * *$ \\
\hline LT & $\mathrm{CIv}$ & .19 & $11.48^{* *}$ & .07 & .02 & .46 & $3.39 * *$ \\
\hline М & $\mathrm{CIv}$ & .13 & $\begin{array}{l}7.61^{*} \\
\text { gresivid }\end{array}$ & $\begin{array}{l}.07 \\
\text { dre }\end{array}$ & .02 & .39 & $2.76^{*}$ \\
\hline LP & $\mathrm{CIv}$ & .20 & $12.36 * *$ & .04 & .01 & .47 & $3.51 * *$ \\
\hline $\mathrm{MP}$ & $\mathrm{CIv}$ & .20 & $11.77 * *$ & .04 & .01 & .46 & $3.43^{* *}$ \\
\hline LT & $\mathrm{CIv}$ & .19 & $11.48^{* *}$ & .07 & .02 & .46 & $3.39 * *$ \\
\hline & $\mathrm{CIV}$ & .13 & $\begin{array}{l}7.61 * \\
\text { etraimie }\end{array}$ & $\begin{array}{r}.07 \\
\text { dre }\end{array}$ & .02 & .39 & $2.76^{*}$ \\
\hline LP & $\mathrm{CIv}$ & .20 & $12.36 * *$ & .04 & .01 & .47 & $3.51 * *$ \\
\hline $1 \mathrm{VII}$ & $\mathrm{CIv}$ & .20 & $11.77 * *$ & .04 & .01 & .46 & $3.43^{* *}$ \\
\hline LT & $\mathrm{CIV}$ & .19 & $11.48^{* *}$ & .07 & .02 & .46 & $3.39 * *$ \\
\hline 101 & $\mathrm{CIv}$ & .13 & $7.61 *$ & .07 & .02 & .39 & $2.76^{*}$ \\
\hline
\end{tabular}




\begin{tabular}{|c|c|c|c|c|c|c|c|}
\hline \multicolumn{8}{|c|}{ Ansiedad Madre } \\
\hline & $\mathrm{CIv}$ & .20 & $12.36^{* *}$ & .04 & .01 & .47 & $3.51^{* *}$ \\
\hline the & $\mathrm{CIv}$ & .20 & $11.77 * *$ & .04 & .01 & .46 & $3.43^{* *}$ \\
\hline & $\mathrm{CIv}$ & .19 & $11.48^{* *}$ & .07 & .02 & .46 & $3.39 * *$ \\
\hline & $\mathrm{CIv}$ & .13 & $7.61 *$ & .07 & .02 & .39 & $2.76^{*}$ \\
\hline
\end{tabular}

En tercer lugar, y siguiendo el mismo procedimiento que en el paso anterior, se llevaron a cabo análisis de regresión jerárquicos para analizar el efecto que las diferentes evaluaciones del ajuste socioemocional manifiestan en las competencias académicas. Comenzando con el ajuste positivo evaluado por el profesor se observa que actúa como predictor de casi todas las medidas de competencia académica (excepto la medida de seguridad). Un perfil de resultados similar se aprecia con la evaluación del ajuste positivo procedente de la madre (véase Tabla 4 para un resumen de los análisis de regresión entre las medidas de ajuste positivo y las medidas de competencias académicas).

Tabla 4. Análisis de regresión entre ajuste positivo (AJP) y competencia académica (SR, RA, SEG, SOC, TF) $(N=49)$.

\begin{tabular}{|c|c|c|c|c|c|c|c|}
\hline Criterio & Modelo & $\mathrm{R}^{2}$ & $F$ & $B$ & SE B & $\beta$ & $t$ \\
\hline \multicolumn{8}{|c|}{ Profesor } \\
\hline \multirow{4}{*}{ RA } & AJP & .47 & $38.99 * *$ & .08 & .01 & .69 & $6.24^{* *}$ \\
\hline & AJP & .51 & $46.62^{* *}$ & .09 & .01 & .72 & $6.82 * *$ \\
\hline & AJP & .60 & $33.56^{* *}$ & .08 & .01 & .66 & $6.73 * *$ \\
\hline & $\mathrm{CIv}$ & & & .03 & .01 & .31 & $3.20^{*}$ \\
\hline SEG & $\mathrm{CIv}$ & .28 & $17.83^{* *}$ & .05 & .01 & .55 & $4.22^{* *}$ \\
\hline \multirow{3}{*}{ TF } & AJP & .73 & $115.58 * *$ & .11 & .01 & .86 & $10.75^{* *}$ \\
\hline & AJP & .56 & $54.86^{* *}$ & .09 & .01 & .75 & $7.41 * *$ \\
\hline & \multicolumn{7}{|c|}{ Madre } \\
\hline \multicolumn{8}{|l|}{ SR } \\
\hline & AJP & .20 & $10.81 * *$ & .26 & .08 & .47 & $3.29 * *$ \\
\hline \multirow{5}{*}{ RA } & AJP & .30 & $9.31 * *$ & .24 & .07 & .43 & $3.15^{*}$ \\
\hline & $\mathrm{CIv}$ & & & .10 & .04 & .34 & 2.51 \\
\hline & $\mathrm{CIv}$ & .16 & 8.64* & .03 & .01 & .43 & $2.94 *$ \\
\hline & $\mathrm{CIv}$ & .27 & $8.19^{* *}$ & .03 & .01 & .38 & $2.79 *$ \\
\hline & AJP & & & .05 & .02 & .35 & $2.55^{*}$ \\
\hline \multicolumn{8}{|c|}{$12.57^{* *}$} \\
\hline \multirow{2}{*}{ TF } & AJP & .19 & $10.22 *$ & .07 & .02 & .46 & $3.20 *$ \\
\hline & AJP & .23 & $12.72 * *$ & .07 & .02 & .50 & $3.57 * *$ \\
\hline
\end{tabular}

Nota: Competencia académica (SR: Seguimiento de reglas, RA: Responsabilidad en el aprendizaje, SEG: Seguridad, SOC: Sociabilidad, TF: Tolerancia a la frustración). Inteligencia (CIv: CI verbal). $*(p<.05),{ }^{* *}(p<.001)$.

Además, en función de la procedencia de la evaluación de los problemas de conducta, se registran efectos diferenciales en las competencias académicas. En el caso de la agresividad, cuando su evaluación procede del profesor, por sí sola y en sentido negativo, actúa como un potente predictor del seguimiento de reglas y la tolerancia a la frustración. También predice la responsabilidad en el aprendizaje y la sociabi- lidad, aunque en ambos casos incorporando en dicha varianza el CI verbal. Sin embargo, cuando la evaluación de la agresividad procede de la madre únicamente predice, en sentido negativo y con valores bajos, la sociabilidad y la tolerancia a la frustración (véase Tabla 5 para un resumen de los análisis de regresión entre las evaluaciones de agresividad y las medidas de competencias académicas). 
Tabla 5. Análisis de regresión entre agresividad (AGR) y competencia académica $(N=49)$.

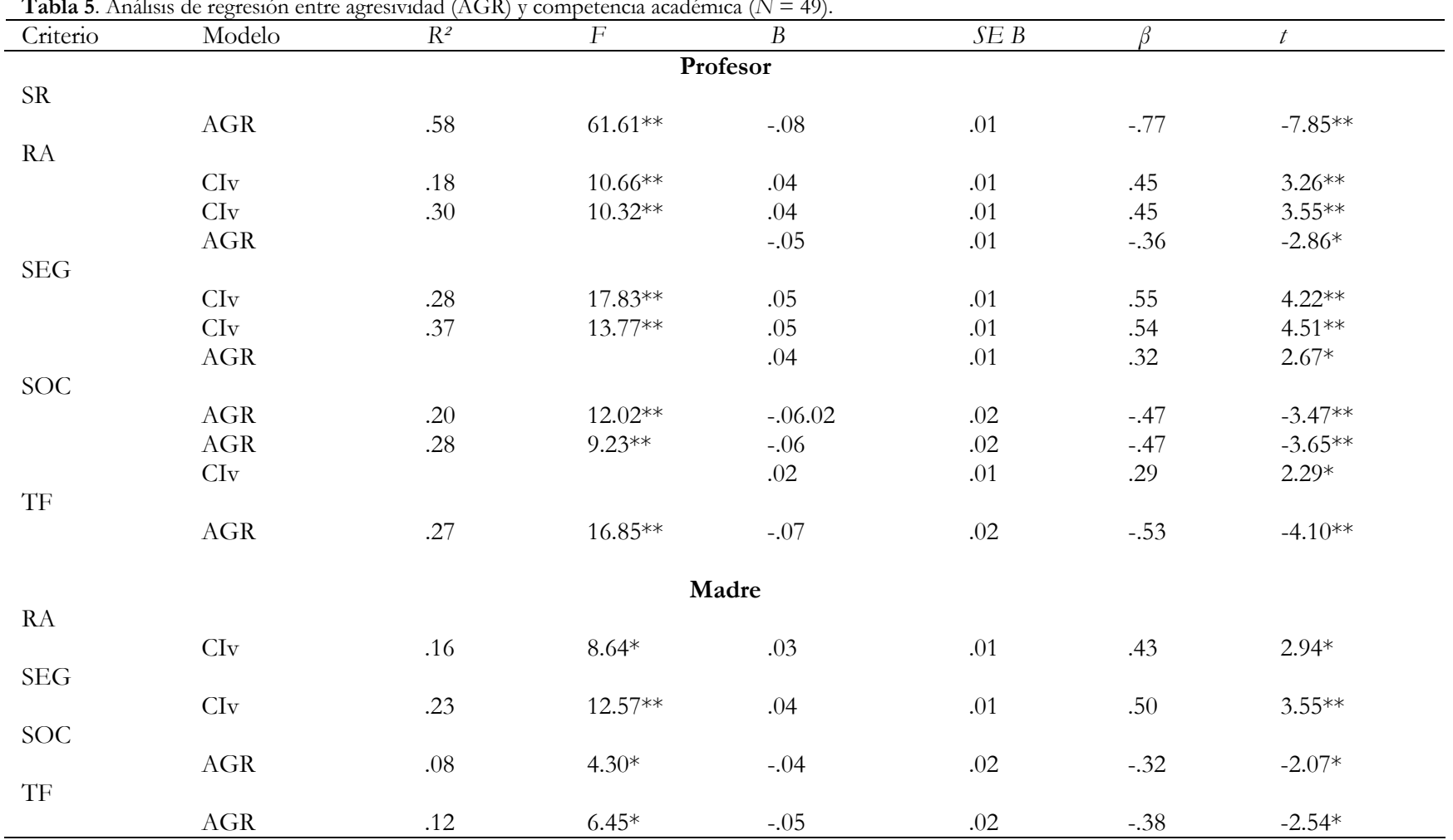

Nota: Competencia académica (SR: Seguimiento de reglas, RA: Responsabilidad en el aprendizaje, SEG: Seguridad, SOC: Sociabilidad, TF: Tolerancia a la frustración). Inteligencia (CIv: CI verbal).

$$
*(p<.05), * *(p<.001)
$$

Cuando el retraimiento lo evalúa el profesor, los resultados señalan que menores valoraciones de retraimiento predicen una mejor sociabilidad. Junto a mayores puntuaciones en el CI verbal se relacionan con mejor responsabilidad en el aprendizaje y mayor seguridad. En cambio, los resultados indican que no tiene ningún valor predictivo para ninguna de las medidas de competencias académicas analizadas, cuando la valoración del retraimiento procede de la madre (véase Tabla 6 para un resumen de los análisis de regresión entre las evaluaciones de retraimiento y las medidas de competencias académicas).

Tabla 6. Análisis de regresión entre retraimiento (RET) y competencia académica $(N=49)$.

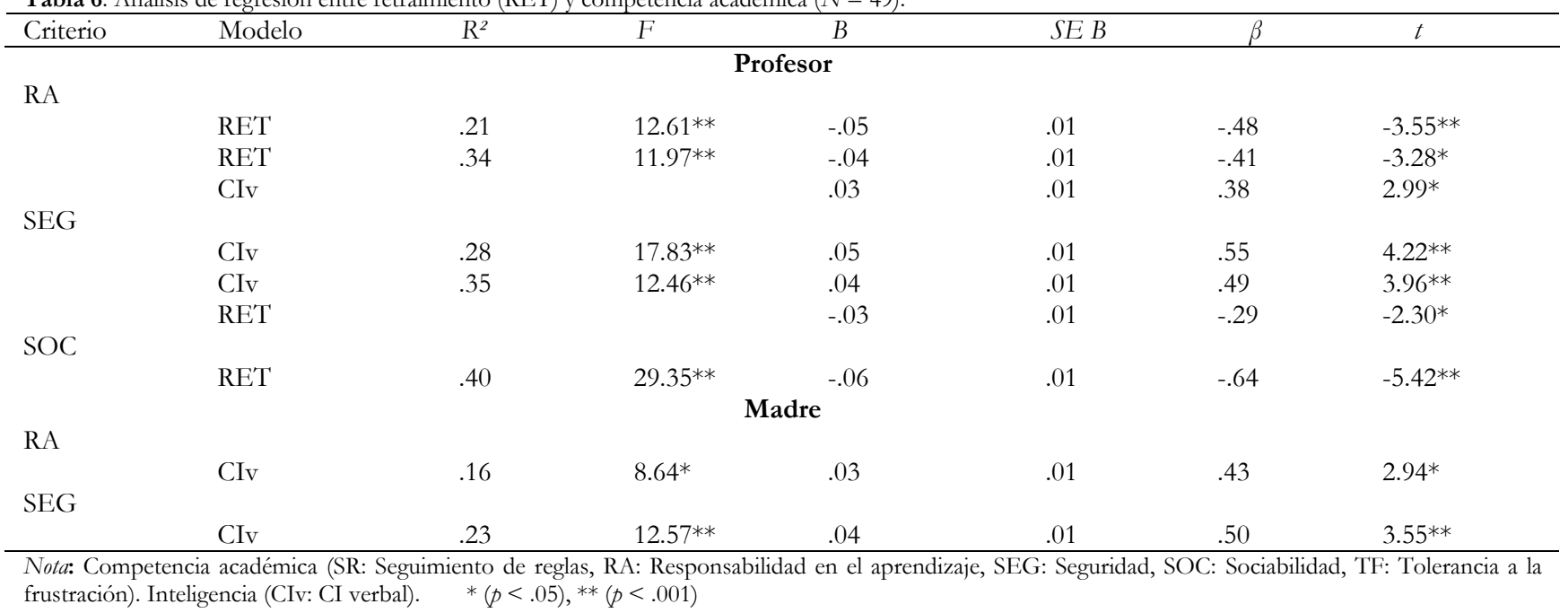


Por último, los resultados obtenidos entre las evaluaciones de ansiedad y las medidas de competencias académicas reflejan el mismo perfil, con independencia de quien evalúe la ansiedad. Sólo predice en sentido negativo el componente de seguridad (véase Tabla 7 para un resumen de los análisis de regresión entre las evaluaciones de ansiedad y las medidas de competencias académicas).

Tabla 7. Análisis de regresión entre ansiedad (ANS) y competencia académica $(N=49)$.

\begin{tabular}{|c|c|c|c|c|c|c|c|}
\hline Criterio & Modelo & $\mathrm{R}^{2}$ & $F$ & $B$ & SE B & $\beta$ & $t$ \\
\hline \multicolumn{8}{|c|}{ Profesor } \\
\hline 121 & CIv & .18 & $10.66^{*}$ & .04 & .01 & .45 & $3.26^{*}$ \\
\hline \multicolumn{8}{|l|}{ SEG } \\
\hline & $\mathrm{CIv}$ & .28 & $17.83^{* *}$ & .05 & .01 & .55 & $4.22^{* *}$ \\
\hline & $\mathrm{CIv}$ & .36 & $13.24 * *$ & .05 & .01 & .55 & $4.64 * *$ \\
\hline & ANS & & & -.06 & .02 & -.31 & $-2.52 *$ \\
\hline \multicolumn{8}{|c|}{ Madre } \\
\hline RA & & & & & & & \\
\hline & $\mathrm{CIv}$ & .16 & $8.64^{*}$ & .03 & .01 & .43 & $2.94^{*}$ \\
\hline \multicolumn{8}{|l|}{ SEG } \\
\hline & $\mathrm{CIv}$ & .23 & $12.57 * *$ & .04 & .01 & .50 & $3.55^{* *}$ \\
\hline & $\mathrm{CIv}$ & .31 & $9.89 * *$ & .03 & .01 & .39 & $2.75^{*}$ \\
\hline & ANS & & & -.06 & .02 & -.33 & $-2.38 *$ \\
\hline
\end{tabular}

Nota: Competencia académica (SR: Seguimiento de reglas, RA: Responsabilidad en el aprendizaje, SEG: Seguridad, SOC: Sociabilidad, TF: Tolerancia a la frustración). Inteligencia (CIv: CI verbal ). * $(p<.05)$.

\section{Discusión}

En respuesta a nuestro primer objetivo, y como se viene recogiendo en investigaciones previas (Achenbach, 2006; Korat, 2004), nuestros resultados reflejan sólo un acuerdo parcial entre las valoraciones que progenitores y profesores tienen del ajuste socioemocional de los niños. Encontramos acuerdo en el ajuste positivo y en la evaluación de los problemas de apatía-retraimiento. Sin embargo, obtenemos discrepancias en las evaluaciones de agresividad y ansiedad, que además no predicen la ejecución escolar del niño de la misma manera. Es decir, aunque encontramos cierta convergencia en las medidas de ajuste socioemocional entre madres y profesores, no predicen de igual forma el comportamiento del niño en el ámbito educativo. Como recientemente han señalado Dirks y colaboradores (2011), estas discrepancias son algo más que un sesgo del evaluador y pueden estar indicando diferentes manifestaciones del comportamiento del niño en función del contexto y la situación en el que se analiza (De los Reyes et al., 2009; Drabick et al., 2008). Además, las diferencias en la naturaleza del contenido de las mismas pueden estar generando en los evaluadores unas expectativas y responsabilidades de ejecución diferentes respecto al niño.

En respuesta al segundo objetivo, al analizar la relación del ajuste socioemocional con el rendimiento académico encontramos importantes divergencias entre profesores y madres. En este sentido, se observa que cuando el ajuste positivo es valorado por el profesor, éste predice el rendimiento que él evalúa, aunque no el evaluado mediante pruebas objetivas. Esto puede interpretarse como que las estimaciones que los profesores realizan del comportamiento de sus alumnos en las situaciones de enseñanza-aprendizaje se relacionan más con las evaluaciones continuas de rendimiento académico, reflejadas en sus calificaciones finales, que con las puntuales de un test (Ray y Elliot, 2006). En este sentido, puede ser que el contenido de los ítems, con los que se evalúa el ajuste socioemocional, sintonice y discrimine acertadamente las expectativas del profesor, en cuanto al tipo de conocimientos y comportamientos que debe tener un buen estudiante en las actividades de enseñanza-aprendizaje. Pero, que dichos contenidos no resulten discriminativos en cuanto al rendimiento evaluado mediante una prueba objetiva. En cambio, cuando se analiza la visión que las madres tienen de la adaptación socioemocional de sus hijos, ésta no se relaciona con ninguna medida de rendimiento académico. Estos resultados van en la línea de los obtenidos por el Instituto Nacional para el Desarrollo y la Salud de los Niños en Estados Unidos (2003), en cuyo informe se señala que, en niños de primer curso de Educación Primaria, la evaluación del ajuste socioemocional que realizan las madres no predice ningún tipo de rendimiento académico. A la luz de lo que se ha expuesto en la introducción, esto podría estar reflejando las diferentes experiencias y expectativas que padres y profesores tienen acerca de las implicaciones que las características evaluadas del ajuste socio-emocional del niño tienen para ajustarse a las demandas que se suscitan en las diferentes situaciones de enseñanza-aprendizaje. De manera que podría interpretarse que para las madres, un buen ajuste social aparece vinculado, por ejemplo, a que el niño tenga amigos y se desarrolle afectivamente y no tanto a las expectativas y cualidades relacionadas con un adecuado rendimiento académico.

Cuando se analizan las evaluaciones de los problemas de ajuste socioemocional con el rendimiento académico, de nuevo aparecen discrepancias entre las evaluaciones de las madres y profesores. Así, mientras que para las madres, ninguno de los comportamientos considerados problemáticos parece tener influencia sobre el rendimiento académico de 
los niños; en cambio, en las evaluaciones que realizan los profesores observamos diferentes perfiles de influencia. Por una parte, hemos podido comprobar cómo la valoración que el profesor realiza de la agresividad se relaciona negativamente con la evaluación del rendimiento en matemáticas. Estos resultados coinciden con otras investigaciones en las que se señala que los niños que manifiestan problemas de comportamiento de tipo externalizante también suelen tener un bajo rendimiento escolar (Jimerson, Egeland, Sroufe y Carlson, 2000). Además, se ha encontrado en investigaciones longitudinales (Campbell, Spieker, Burchinal y Poe, 2006) que de entre los diferentes problemas externalizantes, la agresividad es el que mejor predice un peor funcionamiento académico en el transcurso del tiempo. Una posible explicación puede basarse en la saliencia que tienen las conductas agresivas, que las hace ser fácilmente observables, tanto por los padres como por los maestros, por su importante efecto en las actividades diarias de enseñanza-aprendizaje, interfiriendo el desarrollo de habilidades de trabajo que favorezcan un buen rendimiento académico (McLelland, Morrison y Holmes, 2000).

Por otra parte, encontramos que la evaluación que el profesor realiza de los problemas de apatía-retraimiento, manifiesta una relación negativa con el rendimiento académico en todas las formas analizadas. Esto apoya los resultados de investigaciones que asocian los problemas de tipo internalizante (depresión, introversión, ansiedad) con el fracaso escolar (Ansary y Luthar, 2009; Burt y Roisman, 2010). Y a ello se une, que su valoración de la ansiedad también influye negativamente en el rendimiento, concretamente con la evaluación objetiva en matemáticas. Estos resultados están en consonancia con las relaciones encontradas entre los niveles de estrés y la influencia negativa de la ansiedad sobre el rendimiento escolar y una situación de examen (como el de matemáticas) (Cunningham et al., 2002). A este respecto, el miedo y la ansiedad específicos a las matemáticas se presentan como un impedimento para rendir y afrontar las dificultades que la ejecución de este tipo de evaluaciones conlleva (Beilock, Gunderson, Ramirez y Levine, 2010). No obstante, conviene poner de manifiesto que esas relaciones se producen cuando nos referimos a la evaluación mediante una prueba objetiva y no a la que el profesor realiza como resultado de una evaluación continuada. Son evaluaciones de naturaleza distinta. La evaluación objetiva en sí misma genera una ansiedad muy diferente (ansiedad de test) a la que el alumno puede llegar a tener en las situaciones de enseñanzaaprendizaje de dichos contenidos que son las que en buena parte influyen sobre la evaluación final del profesor.

Respecto a la relación que la valoración del ajuste socioemocional del niño tiene sobre la valoración de sus competencias académicas en el aula, encontramos un mayor acuerdo respecto al ajuste social positivo entre los profesores y las madres, aunque la extensión de dichas relaciones parece ser mayor por parte de los profesores. Cuando la valoración del ajuste positivo la realiza la madre se relaciona positivamente con los comportamientos de seguimiento de reglas, aprendizaje responsable, sociabilidad y tolerancia a la frustración. Así pues, los contenidos que se valoran en el ajuste social del niño parecen guardar una estrecha relación con los contenidos sociales y personales del niño en el contexto educativo, que a su vez, según el informador, se relacionan con el rendimiento académico. Estos resultados están en la línea de los estudios en los que se observa que el efecto del ajuste social sobre el rendimiento no es directo sino que se realiza a través de la influencia que tiene sobre la competencia académica (Ray y Elliot, 2006). Cuando la evaluación del ajuste procede del profesor, se caracteriza por conductas de liderazgo, sensibilidad social y autocontrol, y predice todas las variables de competencias académicas analizadas (seguimiento de reglas, aprendizaje responsable, seguridad, sociabilidad y tolerancia a la frustración). Parece desprenderse de esas predicciones que las interpretaciones del profesor de los contenidos relacionados con el ajuste social del niño guardan una estrecha relación con las expectativas que tiene del comportamiento social del niño en el contexto educativo (Martin y Holbrook, 1985). Desde la perspectiva del docente, se subraya la importancia de la competencia social como un potente indicador del éxito escolar (Burt et al., 2008).

Por otra parte, cuando padres y profesores evalúan las dimensiones relacionadas con los problemas de conducta en el terreno de la competencia académica, de nuevo surgen divergencias entre los evaluadores. En el caso de la evaluación de la agresividad, los profesores a diferencia de las madres, la relacionan con un peor comportamiento general del niño en el aula. En este caso madres y profesores otorgan una significación psicológica muy distinta a un problema claramente observable como es la agresividad. Las atribuciones que realizan los profesores están en consonancia con resultados de investigaciones longitudinales en las que se demuestra el valor predictivo de la agresión sobre un peor funcionamiento académico del niño desde la etapa preescolar hasta los 12 años (Campbell et al., 2006). De nuevo, observamos que las diferencias entre los juicios de padres y profesores residen por un lado, en las distintas experiencias que padres y profesores tienen de la vida social del niño, cuyas manifestaciones, conflictos y resoluciones son diferentes en el hogar y en el colegio y en donde las expectativas de las madres claramente minusvaloran su influencia sobre las competencias académicas.

En relación con la dimensión de apatía-retraimiento de nuevo encontramos valoraciones diferentes entre madres y profesores. Esas diferencias parecen poner de manifiesto que la evaluación de los profesores del retraimiento de sus alumnos guarda una estrecha relación con una valoración negativa del funcionamiento relacionado con el aprendizaje responsable, la sociabilidad y la seguridad. Durante este periodo de edad en el que se encuentran nuestros alumnos las relaciones con los compañeros ejercen una gran influencia en su adaptación social y autoestima. A este respecto, una actitud de pasividad y retraimiento puede tener efectos que no favorecen la dinámica que el profesor desarrolla en el au- 
la y que al mismo tiempo genera-rechazo en sus compañeros (Rubin et al., 2006). El conocimiento de las demandas de contexto educativo y la importancia de la comparación social en la construcción de la individualidad del niño en este periodo, probablemente hayan sido determinantes para las diferentes evaluaciones de madres y profesores, y en este sentido, en la falta de predicciones de las madres de las implicaciones de dicha dimensión en el contexto educativo. De nuevo, parece que las discrepancias entre los evaluadores nos informan de la posible heterogeneidad en cuanto a cómo y a cuánto se manifiesta un problema de conducta en diferentes situaciones. (De los Reyes et al., 2009) En cuanto a la valoración de la dimensión de la ansiedad, tanto padres como profesores coinciden en señalar un mismo perfil de influencia basado en su incidencia sobre el comportamiento asertivo del niño. La poca influencia que se atribuye sobre la competencia académica podría justificarse porque se trata de una muestra no clínica.

En el análisis del efecto del ajuste socioemocional en el rendimiento y las competencias académicas, también se ha controlado la importancia del efecto del CI. En nuestro estudio se pone de manifiesto que ambos aspectos (inteligencia y ajuste socioemocional) son necesarios para explicar el rendimiento y las competencias académicas, ya que en todos los análisis realizados siempre encontramos como predictores el CI junto con algún aspecto del ajuste socioemocional del niño. Como ya se ha señalado, en investigaciones previas se ha puesto de manifiesto que el CI ejerce un efecto directo sobre el funcionamiento académico de los niños y también se observan asociaciones del CI con el ajuste conductual del niño, fundamentalmente con los problemas de conducta y los desórdenes por déficit de atención. Además, se ha encontrado una clara tendencia entre el incremento en el CI y menores índices de problemas de conducta tanto de tipo externalizante como de ansiedad (Fergusson et al., 2005).

A la luz de los datos obtenidos, consideramos acertada la consideración que De los Reyes y colaboradores (De los Re-

\section{Referencias}

Achenbach, T. M. (2006). As others see us: Clinical and research implications of cross-informant correlations for psychopathology. Current Directions in Psychological Science, 15, 94-98.

Alva, S. A., y de los Reyes, R. (1999). Psychosocial stress, internalized symptoms, and the academic achievement of Hispanic adolescents. Journal of Adolescent Research, 14, 343-358.

Ansary, N. S., y Luthar, S. S. (2009). Distress and academic achievement among adolescents of affluence: A study of externalizing and internalizing problem behaviors and school performance. Development and Psychopathology, 21(1), 319-341.

Beilock, S.L., Gunderson, E.A., Ramirez, y Levine, S. (2010). Female teachers' math anxiety affects girls' math achievement. PNAS, 107, 18601863.

Berg-Nielsen, T. S., Solheim, E., Belsky, J., y Wichstrom, L. (2011). Preschoolers' psychosocial problems: In the eyes of the beholder? Adding teacher characteristics as determinants of discrepant parent-teacher reports.Child Psychiatry Human Development. Doi: 10.1007/s10578011-0271-0.

Biederman, J., Faraone, S., Milberger, S., Guite, J., Mick, E., Chen, L., Mennin, D., et al., (1996). A prospective 4-year follow-up study of at- yes et al., 2009; De los Reyes y Kazdin, 2005) de analizar la existencia de discrepancias entre los evaluadores no como una medida de error, sino más bien como un aspecto informativo que nos están indicando que el comportamiento del niño es mucho más diversificado de lo que a priori podría suponerse, mucho más susceptible al contexto que lo desencadena y a los evaluadores que lo interpretan. A ello hay que añadir que cada evaluador informa en función de sus expectativas las cuales a su vez podrían estar influyendo en la dinámica de las relaciones con el niño. Por otro lado, estos resultados sugieren que los contenidos de los ítems y los contextos utilizados para la evaluación del ajuste socioemocional influyen en la interpretación y valoración que tienen los padres y los profesores. A la luz de los datos obtenidos, puede decirse que la valoración del profesor del ajuste socioemocional del niño se construye sobre contenidos sociales relacionados con el contexto educativo, mientras que la de las madres, aunque son buenas informadoras sobre los problemas de conducta de sus hijos, a la hora de valorar su implicación sobre el funcionamiento del niño en el contexto educativo su relevancia parece ser mucho menos acertada que la del profesor. En definitiva, parece que los juicios del profesor están en sintonía con las expectativas que tiene del buen alumno y del rendimiento eficaz. En cambio, la visión de los padres del ajuste socioemocional de sus hijos no se relaciona necesariamente con un buen rendimiento académico.

Finalmente, subrayar la importancia de tener presente el CI de los niños cuando se analiza su funcionamiento escolar, ya que no sólo correlaciona con las medidas de rendimiento y con las de competencia académica, sino que, con independencia de quien evalúe el ajuste socioemocional del niño, el CI predice ambos aspectos del funcionamiento escolar del niño. Además, consideramos de interés profundizar en las relaciones existentes entre ajuste social e inteligencia por el efecto conjunto que han demostrado tener sobre el rendimiento académico de los niños.

tention-deficit hyperactivity and related disorders. Archives of General Psychiatry, 53, 437-446.

Briggs-Gowan, M. J., y Carter, A. S. (2008). Social-emotional screening status in early childhood predicts elementary school outcomes. Pediatrics, 121, 957-962.

Burt, K. B., Obradovic, J., Long, J. D., y Masten, A. S. (2008). The interplay of social competence and psychopathology over 20 years: Testing transactional and cascade models. Child Development, 79(2), 359-374.

Burt, K. B., y Roisman, G. I. (2010). Competence and psychopathology: Cascade effects in the NICHD study of early child care and youth development. Development and Psychopathology, 22(3), 557-567.

Campbell, S. B., Spieker, S., Burchinal, M., y Poe, M. D. (2006). Trajectories of aggression from toddlerhood to age 9 predict academic and social functioning through age 12. Journal of Child Psychology and Psychiatry, 47(8), 791-800.

Campbell, S. B., Shaw, D. S., y Gillion, M. (2000). Early externalizing behavior problems: toddlers and preschoolers at risk for later maladjustment. Developmental Psychopathology, 12(3), 467-488.

Caspi, A., Wright, B. R. E., Moffit, T. E., y Silva, P. A. (1998). Early failure in the labor market: Childhood and adolescent predictors of unem- 
ployment in the transition to adulthood. American Sociological Review, 63, 424-451.

Carreras, M.R., Braza, P., y Braza, F. (2001). Implicación en los comportamientos de agonismo y ajuste socioemocional al grupo de iguales en niños de 4 y 5 años. Psicothema, 13, 258-262.

Coie, J. D., y Dodge, K. A. (1998). Aggression and antisocial behavior. En W. Damon, y N. Eisenberg (Eds.), Handbook of child psychology (pp. 779862). New York: Wiley.

Collishaw, S., Goodman, R., Ford, T., Rabe-Hesketh, S., y Pickles, A. (2009). How far are associations between child, family, and community factors and child psychopathology informant-specific and informant-general? Journal of Child Psychology and Psychiatry, 50, 571-580.

Comer, J. S., y Kendall, P. C. (2004). A symtom-level examination of parentchild agreement in the diagnosis of anxious youth. Journal of the American Academy of Child and Adolescent Psychiatry, 43, 878-886.

Cunningham, M., Hurley, M., Foney, D., y Hayes, D. (2002). Influence of perceived contextual stress on self-esteem and academic outcomes in African American adolescents. Journal of Black Psychology, 28, 215-233.

De los Reyes, A., Henry, D. B., Tolan, P. H., y Wakschlag, L. S. (2009). Linking informant discrepancies to observed variations in young children's disruptive behavior. Journal of Abnormal Child Psychology, 37, 637652.

De los Reyes, A., y Kazdin, A. E. (2005). Informant discrepancies in the assessment of childhood psychopathology: A critical review, theoretical framework, and recomendations for further study. Psychological Bulletin, 131, 483-509.

De los Reyes, A., Youngstrom, E. A., Pabón, S. C., Youngstrom, J. K., Feeny, N. C., y Findling, R. L. (2011). Internal consistency and associated characteristics of informant discrepancies in clinic referred youth age 11 to 17 years. Journal of Clinical Child and Adolescent Psychology, 40(1), 3653.

Demaray, M. K., y Malecki, C. K. (2002). Critical levels of perceived social support associated with student adjustment. School Psychology Quarterly, 17(3), 213-241.

DiPerna, J. C., y Elliot, S. N. (2002). Promoting academic enablers to improve student achievement: An introduction to the mini-series. School Psychology Review, 31, 293-297.

DiPerna, J. C., Volpe, R. J., y Elliot, S. N. (2002). A model of academic enablers and elementary reading/ language arts achievement. School Psychology Review, 31, 298-312.

Dirks, M. A., Boyle, M. H., y Georgiades, K. (2011). Psychological symptoms in youth and later socioeconomic functioning: Do associations vary by informant? Journal of Clinical Child and Adolescent Psychology, 40(1), 10-22.

Dishion, T.J. y Patterson, G.R. (2006). The development and ecology of antisocial behavior in children and adolescents. En D. Cicchetti y D.J. Cohen (Eds.), Developmental psychopathology (2nd ed., Vol. 3, pp. 503-541). New York: Wiley.

Drabick, D. A. G., Gadow, K. D., y Loney, J. (2007). Source-specific oppositional defiant disorder: Comorbidity and risk factors in referred elementary schoolboys. Journal of the American Academy of Child and Adolescent Psychiatry, 46, 92-101.

Drabick, D. A. G., Gadow, K. D., y Loney, J. (2008). Co-occurring ODD and GAD symptom groups: Source-specific syndromes and crossinformant comorbidity. Journal of Clinical Child and Adolescent Psychology, 37, 314-326.

Elliot, S. N., y Gresham, F. M. (2008). Social Skills Improvement System: intervention guide. Minneapolis, MN: Pearson Assessment.

Farrington, D. P. (1995). The development of offending and antisocial behavior from childhood: Key findings from the Cambridge study in delinquent development. Journal of Child Psychology and Psychiatry, 36, 929964.

Ferdinand, R. F., Hoogerheide, K. N., van der Ende, J., Heigmens-Visser, J., Koot, H. M., Kasius, M. C., et al., (2003). The role of the clinician: Three-year predictive value of parents', teachers', and clinicians' judgment of childhood psychopathology. Journal of Child Psychology and Psychiatry, 44, 867-876.

Fergusson, D. M., Horwood, L. J., y Ridder, E. M. (2005). Show me the child at seven II: Childhood intelligence and later outcomes in adoles- cence and young adulthood. Journal of Child Psychology and Psychiatry, 46(8), 850-858

Foley, D. L., Rutter, M., Angold, A., Pickles, A., Maes, H. M., Silberg, J. L., et al., (2005). Making sense of informant disagreement for overanxious disorder. Journal of Anxiety Disorders, 19(2), 193-210.

Frank, S. J., Van Egeren, L. A., Fortier, J. L., y Chase, P. (2000). Structural, relative, and absolute agreement between parents' and adolescent impatients' reports of adolescent functional impairment. Journal of $A b-$ normal Child Psychology, 28(4), 395-402.

Galaif, E., Sussman, S., Chou, C. P., y Wills, T. (2003). Longitudinal relations among depression, stress and coping in high risk youth. Journal of Youth and Adolescence, 32, 243-258.

Gazelle, H., y Ladd, G.W. (2003). Anxious solitude and peer exclusión: A diathesis-stress model of intenalizing trajectories in childhood. Child Development, 74, 257-278.

Goodman, R., Simonoff, E., y Stevenson, J. (1995). The impact of child IQ, parent IQ and sibling IQ on child behavioral deviance scores. Journal of Child Psychology and Psychiatry, 36, 409-425.

Gresham, F. M., Elliot, S. N., Cook, C. R., Vance, M. J., y Kettler, R. (2010). Cross-informant agreement for ratings for social skill and problem behavior ratings: An investigation of the Social Skills Improvement System-Rating Scales. Psychological Assessment, 22, 157-166.

Guion, K., Mrug, S., y Windle, M. (2009). Predictive value of informant discrepancies in reports of parenting: Relations to early adolescents' adjustment. Journal of Abnormal Child Psychopathology, 37, 17-30.

Hartley, A. G., Zakriski, A. L., y Wright, J. C. (2011). Probing the depths of informant discrepancies: Contextual influences on divergence and convergence. Journal of Clinical Child and Adolescent Psychology, 40(1), 54-66.

Hinshaw, S. P., y Lee, S. (2003). Conduct and oppositional defiant disorders. En R. A. Barkley y E. J. Mash (Eds.), Child psychopathology (2nd ed., pp. 144-198). New York: Guilford.

Jensen, A. R. (1998).The G factor: The science of mental ability. Westport, CT: Greenwood.

Jimerson, S., Egeland, L., Sroufe, A., y Carlson, B. (2000). A prospective study of high school dropouts examining multiple predictors across development. Journal of School Psychology, 38, 525-549.

Karver, M. S. (2006). Determinants of multiple informant agreement on child and adolescent behavior. Journal of Abnormal Child Psychology, 34(2), 251-262.

Kemper, T. S., Gerhardstein, R., Repper, K. K., y Kistner, J. A. (2003). Mother-child agreement on reports of internalizing symptoms among children referred for evaluation of ADHD. Journal of Psychopathology and Behavioral Assessment, 25, 239-250.

Keogh, B. K., Juvonen, J., y Bernheimer, L. P. (1989). Assessing children's competence: Mothers' teachers' ratings of competent behavior. Psychological Assessment, 1, 224-229.

Korat, O. (2004). Mothers' and teachers' attributions of the academic functioning of Israeli second graders: a comparison between social groups. Early Childhood Research Quarterly, 19, 485-501.

Kraemer, H. C., Measelle, J. R., Ablow, J. C., Essex, M. J., Boyce, W. T., y Kupfer, D. J. (2003). A new approach to integrating data from multiple informants in psychiatric assessment and research: Mixing and matching contexts and perspectives. American Journal of Psychiatry, 160, 15661577.

Martin, R. P., y Holbrook, J. (1985). Relationship of temperament characteristics to the academic achievement of first-grade children. Journal of Psychoeducational Assessment, 3, 131-140.

Masten, A. S., Burt, K. B., y Coatsworth, J. D. (2006). Competence and psychopathology in development. En D. Cicchetti y D.J. Cohen (Eds.), Developmental psychopathology (2nd ed., Vol. 3, pp. 696-738). New York: Wiley.

Masten, A. S., Roisman, G. I., Long, J. D., Burt, K. B., Obradovick, J., Riley, J. R., Boelcke-Stennes, K., y Tellegen, A. (2005). Developmental cascades: Linking academic achievement and externalizing and internalizing symptoms over 20 years. Developmental Psychology, 41(5), 733-746.

McLelland, M. M., Morrison, F. J., y Holmes, D. L. (2000). Children at risk for early academic problems: the role of learning related skills. Early Childhood Research Quarterly, 15, 307-329.

Nakamoto, J. y Schwartz, D. (2010). Is peer victimization associated with academic achievement? A meta-analytic review. Social Development, 19, 221-242. 
National Institute of Child Health and Human Development Early Child Care Research Network (2003). Social functioning in first grade: associations with earlier home and child care predictors and with current classroom experiences. Child Development, 74(6), 1639-1662.

Palmen, H., Vermande, M. M., Dekovic, M., y van Aken, M. A. G. (2011). Competence, problema behavior, and the effects of having no friends, aggressive friends, or nonaggressive friends: A four-year longitudinal study. Merrill Palmer Quarterly, 57(2), 186-213.

Parker, J. G., Rubin, K. H., Erath, S. A., Wojslawowicz, J. C., y Buskirk, A. A. (2006). Peer relationships, child development, and adjustment: A developmental psypathology perspective. En D. Cicchetti y D.J. Cohen (Eds.), Developmental psychopathology (2nd ed., Vol. 1, pp. 419-493). New York: Wiley.

Preddy, T. M., y Fite, P. J. (2012). Differential associations between relation$\mathrm{al}$ and overt aggression and children's psychosocial adjustment. Journal of Psychopathology and Behavioral Assessment. Doi: 10.107/s10862-0119274-1.

Putallaz, M., Grimes, C. L., Foster, K. J., Kupersmidt, J. B., Coie, J. D., y Dearing, K. (2007). Overt and relational aggression and victimization: multiple perspectives within the school setting. Journal of School Psychology, 45, 523-547.

Rapport, M. D., Scanlan, S. W., y Denney, C. B. (1999). Attentiondeficit/hyperactivity disorder and scholastic achievement: A model of dual developmental pathways. Journal of Child Psychology and Psychiatry, 40, 1169-1183.
Ray, C. E., y Elliot, S. N. (2006). Social adjustment and academic achievement: A predictive model for student with diverse academic and behavior competencies. School Psychology Review, 35(3), 493-501.

Rubin, K. H., Bukowski, W. M, y Parker, J.G. (2006). Peer interactions, relationships, and groups. En W. Damon, R. M. Lerner, y N. Eisenberg (Eds.), Handbook of child psychogy (6 $6^{\text {th }}$ ed., Vol. 3, pp. 571-645). New York: Wiley.

Sandoval, M., Lemos, S., y Vallejo, G. (2006). Competences and problems in Spanish adolescents: A normative study of the YSR. Psicothema, 18, 804809.

Schwartz, D., Gorman, A. H., Dodge, K. A., Pettit, G. S., y Bates, J. E. (2008). Friendships with peers who are low or high in aggression as moderators of the link between peer victimization and declines in academic functioning. Journal of Abnormal Child Psychology, 36, 719-730.

Silva, F. y Martorell, M. C. (1999). BAS 1-2. Batería de socialización para profesores y padres (3a. ed.). Madrid: TEA.

Tu, K. M., Erath, S. A., y Flanagan, K. S. (2012). Can socially adept friends protect peer-victimized early adolescents against lower academic competence? Journal of Applied Developmental Psychology, 33, 24-30.

Wechsler, D. (2000). WISC-R. Escala de Inteligencia Wechsler para niñosRevisada. (7ª . ed.). Madrid: TEA.

Zubeidat, I., Fernández-Parra, A., Ortega, J., Vallejo, M. A., Sierra, J. C. (2009). Características psicosociales y psicopatológicas en una muestra de adolescentes españoles a partir del Youth Self-Report/11-18. Anales de Psicología, 25(1), 60-69.

(Artículo recibido: 17-06-2011, revisado: 18-01-2012, aceptado: 23-02-2012) 